\title{
Clinical Outcomes of Aberration-Free All Surface Laser Ablation (ASLA) vs. Aberration-Free ASLA Assisted by Smart Pulse Technology in High Myopia: A One-Year Follow-Up Study
}

\author{
XiaoHao Du $\mathbb{D},{ }^{1,2}$ Jia Zhang, ${ }^{1,3}$ Meng Su, ${ }^{1}$ WenJia Cao, ${ }^{1}$ Shuang Zeng, ${ }^{1}$ QinMei Wang, \\ Ioannis M. Aslanides, ${ }^{4}$ and ShiHao Chen $\rrbracket^{1,3,5}$ \\ ${ }^{1}$ School of Ophthalmology and Optometry, Wenzhou Medical University, Wenzhou, Zhejiang, China \\ ${ }^{2}$ Henan Provincial People's Hospital, Henan Provincial Eye Hospital, Zhengzhou, Henan, China \\ ${ }^{3}$ Eye Hospital of Wenzhou Medical University, Wenzhou, Zhejiang, China \\ ${ }^{4}$ Emmetropia Mediterranean Eye Institute, Heraklion, Crete, Greece \\ ${ }^{5}$ Taizhou Eye Hospital, Taizhou, Zhejiang, China
}

Correspondence should be addressed to ShiHao Chen; chenle@rocketmail.com

Received 4 July 2021; Revised 25 August 2021; Accepted 6 September 2021; Published 18 October 2021

Academic Editor: Zisis Gatzioufas

Copyright (C) 2021 XiaoHao Du et al. This is an open access article distributed under the Creative Commons Attribution License, which permits unrestricted use, distribution, and reproduction in any medium, provided the original work is properly cited.

Purpose. To compare the clinical outcomes of aberration-free all surface laser ablation (ASLA) with and without the use of smart pulse technology (SPT) in high myopia. Methods. This study retrospectively analyzed 138 eyes (138 patients, only the right eye was selected) treated for high myopia (spherical equivalent $\geq-6.00$ diopters) using aberration-free ASLA (non-SPT group; 85 eyes) and aberration-free ASLA assisted by SPT (SPT group; 53 eyes). Examinations such as visual acuity, refraction, and haze were performed before the 12-month follow-up. Corneal epithelial healing time was assessed in the first postoperative day. Visual acuity and refraction examination were performed at 7 days and 1, 3, 6, and 12 months postoperatively. Corneal haze was evaluated in 1 , 3, 6, and 12 months. Safety, efficacy, and corneal wavefront aberrations were assessed 12 months after the treatment. Results. At 12 months postoperatively, $60 \%$ versus $40 \%$ of eyes achieved $20 / 16$ Snellen lines or better, and $92 \%$ versus $82 \%$ of eyes achieved $20 / 20$ Snellen lines or better visual acuity in the SPT and the non-SPT groups, respectively. The average postoperative epithelial healing time was $3.75 \pm 1.00$ days in the SPT group and $3.73 \pm 1.30$ days in the non-SPT group $(P \geq 0.05)$. The safety and the efficacy index of the SPT group were better than those of the non-SPT group in the follow-ups. The attempted spherical equivalent before the surgery and the achieved spherical equivalent at 12 months were comparable between the two groups. Regarding the aberrations, the results of Coma $90^{\circ}$ in the SPT group were better than those in the non-SPT group $(P \leq 0.05)$, but the increase of RMS HOAs (root mean square higher order aberrations), Coma $0^{\circ}$, and spherical aberration postoperatively had no statistical difference between the two groups $(P \geq 0.05)$. Conclusions: Both aberration-free ASLA with and without SPT showed favorable safety, effectiveness, and predictability within 12 months for high myopia. And, ASLA using SPT might have potential advantages in the long-term visual quality.

\section{Introduction}

The term transepithelial photorefractive keratectomy (TransPRK) was firstly introduced by Alio in 1993s [1]. Differing from the chemical or mechanical methods used in earlier conventional surface surgery, TransPRK was a twostep process [2], including epithelial removal followed by stromal ablation. Subsequently, a series of improvements were followed such as one-step TransPRK, reverse one-step TransPRK, and smart pulse technology-assisted TransPRK [3-7]. Due to the quick vision recovery, the latest TransPRK approach has gradually been widely recommended by clinicians $[5,8]$.

In 2014, a new laser ablating mode using a fullerene threedimensional model, referred to as smart pulse technology (SPT), was introduced. It is available on the SCHWIND 
Amaris platform (SCHWIND eye-tech-solutions $\mathrm{GmbH}$, Kleinostheim, Germany) termed transepithelial all surface laser ablation (ASLA) and replaced the original method of TransPRK based on a grid-like corneal representation mode by software update. The advantages of SPT-assisted ASLA (SPT-ASLA) such as early postoperative vision recovery, pain and epithelial healing, corneal asphericity, and reduction of the irregularity of the stromal surface have been reported in some studies $[6,9,10]$. The results of the available research indicate that SPT-ASLA might optimize the geometric arrangement of pulses during laser ablation to make the corneal bed smoother, leading to earlier postoperative visual recovery, faster corneal re-epithelialization, and less haze occurrence than surface ablation without the SPT. However, there are few studies on the difference between SPT-ASLA and non-SPTASLA in the correction effect of high myopia. In this study, we aim to compare the results of those two ablation modes in the correction of high myopia.

The aim of this study was to comparatively evaluate the long-term clinical outcomes of aberration-free SPT-ASLA versus non-SPT-ASLA in patients with high myopia.

\section{Materials and Methods}

2.1. Patients. This comparative observational case series study was to retrospectively analyze 138 eyes (138 patients, only the right eye was selected) which were included in two groups of SPT-ASLA (SPT group; 53 eyes) and non-SPTASLA (non-SPT group; 85 eyes) and compare their longterm efficacy and safety. The cohorts of both groups comprised patients with high myopia who underwent SPT-ASLA or non-SPT-ASLA treatment at the department of refractive surgery of Eye Hospital, WMU, from 2013 to 2016 and met the screening criteria. The inclusion criteria included spherical equivalent of more than -6.00 diopters (D), age more than 18 years, stable refraction for at least 1 year, and the washout period for soft contact lens, hard contact lens, and orthokeratology was more than 2 weeks, 4 weeks, and 12 weeks, respectively. The exclusion criteria included keratoconus or abnormal corneal topography, previous history of intraocular or corneal surgery, ocular infection or active inflammation, autoimmune diseases, and pregnant or nursing women.

This study was reviewed and approved by the ethics committee of the Eye Hospital, WMU (Ethical approval number: 2019-197-k-177). Each patient was informed about the purpose and the procedure of this study, and informed consent was obtained before surgery.

\subsection{Preoperative and Postoperative Assessment. All patients} received detailed ophthalmological examination before surgery including uncorrected distance visual acuity (UDVA), corrected distance visual acuity (CDVA), refraction (manifest and cycloplegic refraction were performed by an optometrist), autorefractometry (RT-5100, NIDEK, Japan) such as sphere (Sph), cylinder (Cyl), and spherical equivalent (SE), slit lamp examination (SL-2G; TOPCON, Japan), and Scheimpflug-based corneal topography (Pentacam HR; Oculus Optikgeräte GmbH, Wetzlar, Germany). Corneal wavefront aberrations were also measured using Pentacam.

All patients of the study were followed up for 7 days and 1, 3, 6, and 12 months postoperatively. The parameters included the corneal epithelial healing time, visual acuity, refraction, safety, efficacy, corneal haze (Fantes scale) [11], and corneal wavefront aberrations.

2.3. Surgical Procedure. Before surgery, each patient received oral vitamin $\mathrm{C}$ tablets twice a day for one week, artificial tears four times a day for three days, and $0.5 \%$ levofloxacin four times a day for two days. Prednisone tablets $(30 \mathrm{mg})$ were taken on the morning of the day before surgery, the day of surgery, and the day after surgery.

Regarding the surgical procedure itself, after conventional disinfection of the conjunctiva and the eyelids, an eye speculum was used to expose the surgical area and alcaine eye drops were administered for surface anesthesia. Aberration-free treatment was performed by the AMARIS 750S excimer laser platform (SCHWIND eyetech-solutions $\mathrm{GmbH}$, Kleinostheim, Germany) with a single continuous profile. Both groups were treated using aberration-free profiles. In the first group, the SPT update was not installed yet (non-SPT-ASLA), while in the second one, the treatments were performed with the SPT installed (SPT-ASLA). After the laser ablation, $0.02 \%$ mitomycin- $C$ was applied to infiltrate the substrate bed, and the eye was then irrigated with cold balanced salt solution. $0.5 \%$ tobramycin dexamethasone was applied on the stromal bed, and a bandage contact lens (Air Optix Night\&Day Aqua Soft Contact Lenses; Alcon Laboratories, America) was applied on the cornea until the epithelial healing was complete.

After surgery, $0.5 \%$ tobramycin dexamethasone eye drops were applied every 2 hours on the day of the surgery and then four times a day for 1 week. The dosage was adjusted appropriately according to the corneal condition. 1 month postoperatively, it was changed to $0.1 \%$ fluorometholone eye drops three times a day and then gradually decreased to 1 drop/month until drug withdrawal for about 4 months after surgery. Artificial tears were applied once an hour for 3 days after the operation and then changed to four times a day for 6 months. Oral vitamin $C$ tablets were taken $500 \mathrm{mg}$ twice a day for two weeks postoperatively.

2.4. Statistical Analysis. The values are expressed as mean\pm standard deviation (SD). The differences between preoperative and postoperative outcomes at 12 months were calculated using the paired Student's $t$-test or the Wilcoxon test, as needed. The group $t$-test or the Mann-Whitney $U$ test were used to compare the differences between the two groups, as needed. Repeated measures analysis of variance was applied to the data of multiple measurements. A $P$ value of 0.05 or less was considered statistically significant. All analyses were performed by SPSS software (version 23; IBM Corporation, Armonk, NY). 


\section{Results}

This study includes 53 eyes in the SPT group and 85 eyes in the non-SPT group. The preoperative baseline of the two groups is shown in Table 1.

3.1. Healing Time of Corneal Epithelium. The mean postoperative healing time of the corneal epithelium was $3.75 \pm 1.00$ days and $3.73 \pm 1.30$ days in the SPT and the nonSPT group, respectively $(P=0.399)$. Epithelial healing was observed at 3 days after treatment in most eyes of both groups. The healing process was completed in 3 to 6 days after surgery in the SPT group and in up to 10 days in the non-SPT group (Figure 1).

3.2. Visual Acuity and Refraction. At 12 months after the treatment, the change of UDVA, sphere, cylinder, and spherical equivalent has no statistical difference between two groups. The improvement of CDVA in SPT group seems statistically better than that in the non-SPT group (Table 2).

3.3. Safety and Efficacy. The distribution diagram of UDVA in 12 months postoperative is shown in Figure 2(a). There were $60 \%$ of eyes and $40 \%$ of eyes which achieved 20/16 (Snellen lines) or better in the SPT group and in the non-SPT group, respectively. In addition, there were $92 \%$ and $82 \%$ of the eyes which achieved 20/20 (Snellen lines) or better in the SPT and non-SPT group, respectively. Regarding CDVA 12 months postoperatively, $2 \%$ of eyes in the SPT group and $11 \%$ of the eyes in the non-SPT group had lost up to 1 Snellen lines, but there were no eyes with more than 2 lines lost (Figure 2(b)). The safety index (postoperative mean CDVA/ preoperative mean CDVA) and the efficacy index (postoperative mean UDVA/preoperative mean CDVA) of the SPT group were statistically better than those of the non-SPT group (Table 3).

3.4. Accuracy and Stability. Attempted spherical equivalent before surgery was $-8.00 \pm 1.30$ diopter (D) in the SPT group, which was not different compared to the one in the non-SPT group $(-8.10 \pm 1.04 \mathrm{D}, P=0.456)$. The achieved spherical equivalent at 12 months after the surgery was $-8.01 \pm 1.41 \mathrm{D}$ in the SPT group, which was not statistically different to the one in the non-SPT group $(-8.15 \pm 1.15 \mathrm{D}$, $P=0.493)$. The predictive curve of refraction of the two groups had similar linear relationships ((c1) and (c2) in Figure 2(c)). 12 months postoperatively, $85 \%$ of the eyes in the SPT group were within $0.50 \mathrm{D}$ of emmetropia, whereas in the non-STP group, $79 \%$ of the eyes were within $0.50 \mathrm{D}$ of emmetropia $(P=0.107)$ (Figure $2(\mathrm{~d}))$.

The refractive stability of both groups (SPT and non$\mathrm{SPT}$ ) is shown in (e1) and (e2) in Figure 2(e), respectively.

3.5. Haze. The occurrence rate of haze is shown in Figure 3. The haze score was similar in both groups $(P>0.05)$. More than $20 \%$ of eyes developed mild haze in both groups at 1 month and 3 months after surgery, and most of them were 0.5 grade (haze traces) and were decreased gradually during the follow-up of the next month.

3.6. Corneal Aberrations. The post-12-month root mean square higher order aberrations (RMS HOA) in the two groups were statistically increased than those in the preoperative stage $(P<0.001)$ (Table 4$)$. The values of spherical aberration and the horizontal coma (Coma $\left.0^{\circ}\right)$ in the two groups were increased postoperatively $(P<0.001)$, but there was no statistical difference between the SPT group and the non-SPT group. The values of the postoperative vertical coma (Coma $90^{\circ}$ ) in the two groups were statistically increased than preoperatively $(P<0.05)$. Nevertheless, the increase was smaller in the SPT group $(P=0.003)$.

\section{Discussion}

Safety, efficacy, and predictability of TransPRK have been proved by several previous studies [12-16]. The application of smart pulse technology (SPT) in all surface laser ablation (ASLA) causes better early postoperative visual recovery and faster epithelial healing [6]. This study mainly compared the long-term results of aberration-free ASLA with and without the use of SPT for high myopia to make sure which might have better outcomes in high myopia.

Serrao and Lombardo [17] showed that the more regular the surface of the corneal stroma after PRK was, the faster the healing of the corneal epithelial would be. Meanwhile, the postoperative healing of the epithelium in eyes with high myopia was significantly slower than in cases with low myopia. SPT uses three-dimensional model of fullerene to optimize the geometric arrangement of pulses during laser ablation, which theoretically provides a smoother corneal stromal bed and faster healing of the corneal epithelium. Another study by Aslanides [6] showed the outcomes of 89 eyes (49 eyes in the SPT group, mean spherical equivalent: $-3.87 \pm 2.21 \mathrm{D} ; 40$ eyes in the non-SPT group, mean spherical equivalent: $-4.8 \pm 1.86 \mathrm{D}$ ). Their results of the epithelial healing time showed that all of the eyes were healed within 3 days in both groups, and it was better in the SPT group than in the non-SPT group. However, in our study, there was no statistically significant difference in mean epithelial healing time between the two groups, and the healing process took longer. The reason might be that the limitation of our study was patients with high myopia (mean spherical equivalent $>-6.00 \mathrm{D}$ ) and a relatively long time was spent for corneal epithelial healing and remodeling, which might weaken the effects of the SPT in making the ablation surface smoother and easier to heal the epithelium. Meanwhile, the range of treatment areas was also a factor affecting the results of the study.

Almost all the results of the safety index and the efficacy index were also superior in the SPT group. Previous studies have shown that the smoothness of regenerated epithelium strongly depends on the roughness of the residual corneal stromal surface [18-21]. If the corneal epithelial cells regenerated and migrated in a smoother plane, the 
TABLE 1: Baseline preoperative values ${ }^{\mathrm{a}}$.

\begin{tabular}{|c|c|c|c|}
\hline Baseline preoperative values & SPT group & Non-SPT group & $P$ \\
\hline Number of eyes & 53 & 85 & \\
\hline Mean age (years) & $26.25 \pm 5.64$ & $24.62 \pm 4.91$ & 0.082 \\
\hline Mean preoperative logMAR UDVA & $1.294 \pm 0.245$ & $1.280 \pm 0.269$ & 0.725 \\
\hline Mean preoperative logMAR CDVA & $-0.007 \pm 0.017$ & $-0.024 \pm 0.034$ & $0.02^{\mathrm{b}}$ \\
\hline \multicolumn{4}{|l|}{ Mean preoperative refractive errors (D) } \\
\hline Sphere & $-7.60 \pm 1.31$ & $-7.60 \pm 1.06$ & 0.925 \\
\hline Cylinder & $-0.83 \pm 0.64$ & $-1.00 \pm 0.71$ & 0.206 \\
\hline Spherical equivalent & $-8.02 \pm 1.32$ & $-8.10 \pm 1.05$ & 0.507 \\
\hline Optical zone diameter $(\mathrm{mm})$ & $6.12 \pm 0.26$ & $6.18 \pm 0.26$ & 0.494 \\
\hline Treatment diameter $(\mathrm{mm})$ & $7.91 \pm 0.33$ & $8.09 \pm 0.33$ & $0.001^{\mathrm{b}}$ \\
\hline Ablation depth $(\mu \mathrm{m})$ & $160.85 \pm 13.05$ & $171.49 \pm 11.25$ & $<0.001^{\mathrm{b}}$ \\
\hline Residual stromal bed thickness $(\mu \mathrm{m})$ & $345.83 \pm 21.44$ & $330.11 \pm 18.71$ & $<0.001^{\mathrm{b}}$ \\
\hline
\end{tabular}

$\mathrm{SPT}=$ smart pulse technology; UDVA = uncorrected distance visual acuity; CDVA=corrected distance visual acuity. ${ }^{\text {a }}$ Values are presented as mean\pm standard deviation. ${ }^{\mathrm{b}}$ Significant difference between the SPT and non-SPT groups (Student's $t$-test/Mann-Whitney $U$-test).

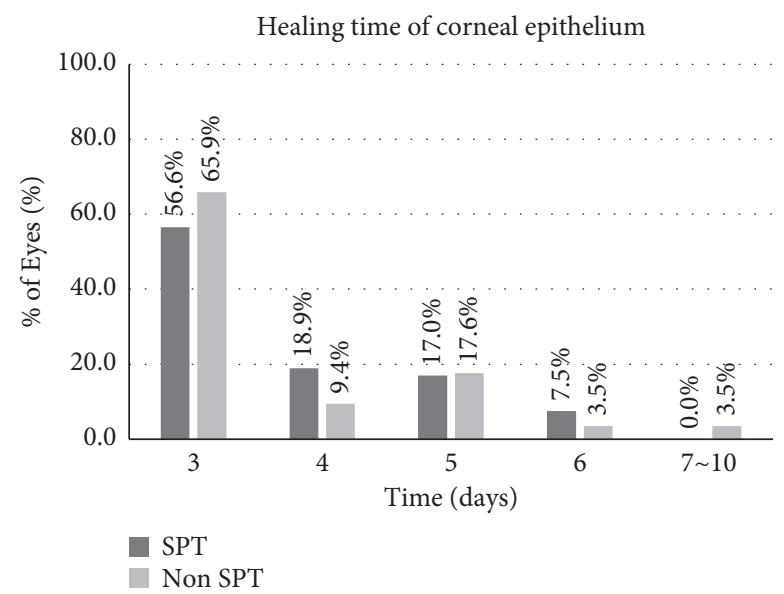

FIGURe 1: Postoperative healing time of corneal epithelium in the SPT and the non-SPT groups. SPT =smart pulse technology.

TABLE 2: Visual acuity and refraction outcomes ${ }^{\mathrm{a}}$.

\begin{tabular}{|c|c|c|c|c|}
\hline Parameter & Group & Preoperative & 12 months & $P$ \\
\hline LogMAR UDVA & $\begin{array}{c}\text { SPT } \\
\text { Non-SPT }\end{array}$ & $\begin{array}{l}1.294 \pm 0.245 \\
1.280 \pm 0.269\end{array}$ & $\begin{array}{l}-0.040 \pm 0.054 \\
-0.009 \pm 0.080\end{array}$ & 0.533 \\
\hline LogMAR CDVA & $\begin{array}{c}\text { SPT } \\
\text { Non-SPT }\end{array}$ & $\begin{array}{l}-0.007 \pm 0.017 \\
-0.024 \pm 0.034 \\
\end{array}$ & $\begin{array}{l}-0.046 \pm 0.045 \\
-0.038 \pm 0.041 \\
\end{array}$ & $0.004^{\mathrm{b}}$ \\
\hline Sphere & $\begin{array}{c}\text { SPT } \\
\text { Non-SPT }\end{array}$ & $\begin{array}{l}-7.60 \pm 1.31 \\
-7.60 \pm 1.06\end{array}$ & $\begin{array}{l}0.24 \pm 0.50 \\
0.26 \pm 0.51\end{array}$ & 0.770 \\
\hline Cylinder & $\begin{array}{c}\text { SPT } \\
\text { Non-SPT }\end{array}$ & $\begin{array}{l}-0.83 \pm 0.64 \\
-1.00 \pm 0.71\end{array}$ & $\begin{array}{l}-0.45 \pm 0.34 \\
-0.42 \pm 0.28\end{array}$ & 0.169 \\
\hline Spherical equivalent & $\begin{array}{c}\text { SPT } \\
\text { Non-SPT }\end{array}$ & $\begin{array}{l}-8.02 \pm 1.32 \\
-8.10 \pm 1.05 \\
\end{array}$ & $\begin{array}{l}0.01 \pm 0.47 \\
0.05 \pm 0.50\end{array}$ & 0.493 \\
\hline
\end{tabular}

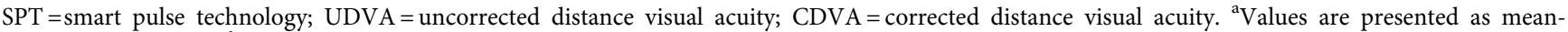
\pm standard deviation. ${ }^{b}$ Significant difference between the SPT and non-SPT groups (Student's $t$-test/Mann-Whitney $U$-test).

connections between the cells are also more regular. In addition, the fibroblast proliferation and corneal opacity would be reduced, which may improve the visual quality to some extent and thus affect the postoperative eyesight. This fact could explain the better results in the SPT group.

As stated in previous studies, surgical accuracy becomes more elusive as the degree of correction increases. [21, 22]
Meanwhile, the deeper the ablation, the more the hyperplasia of the epithelium, and the hyperplasia of corneal epithelium is associated with refractive regression [23]. This may indicate that the prediction of long-term postoperative refractive results of high myopia is more difficult than that of low to moderate myopia. In our study, the predictive curves drawn by the follow-up results of the two groups after 1 year 

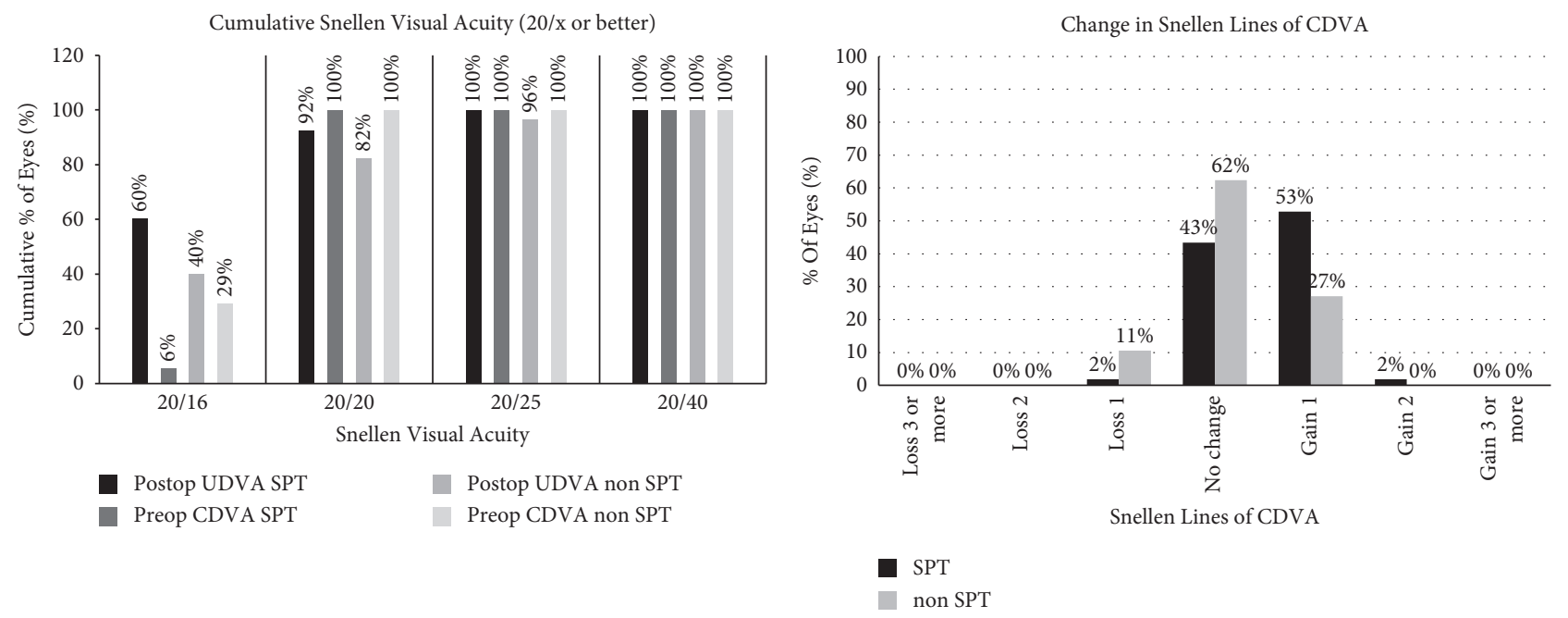

(a)

(b)
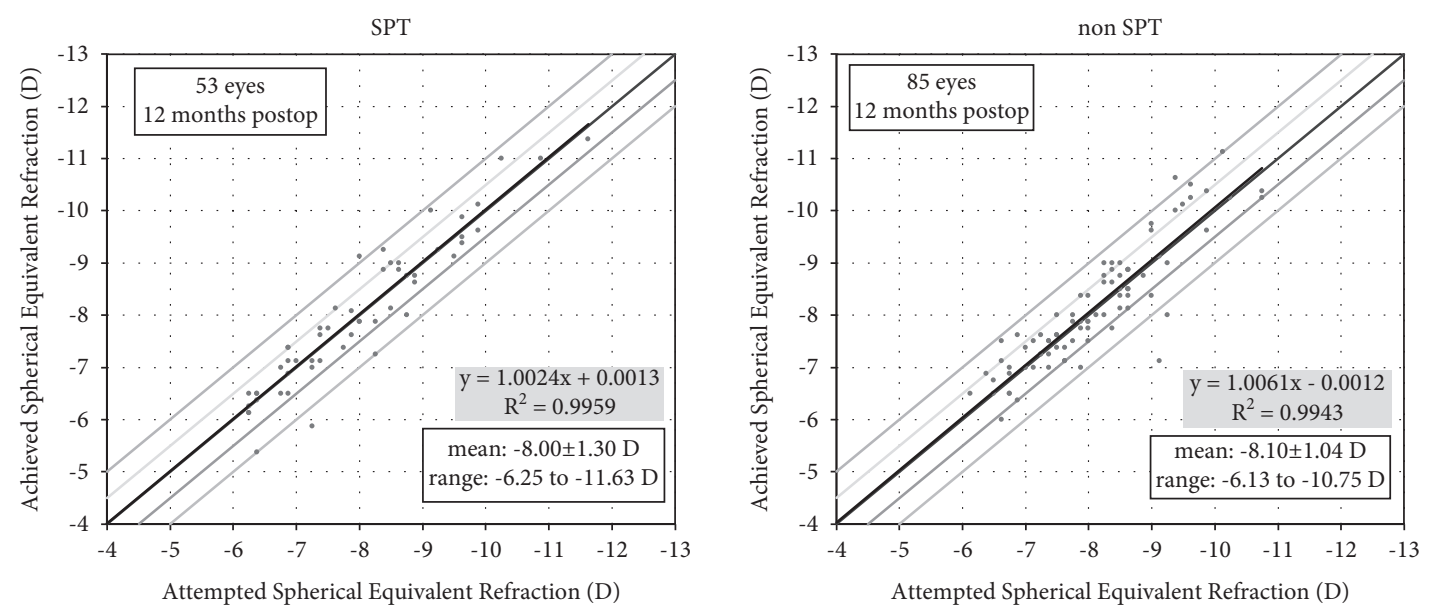

(c2)

(c)

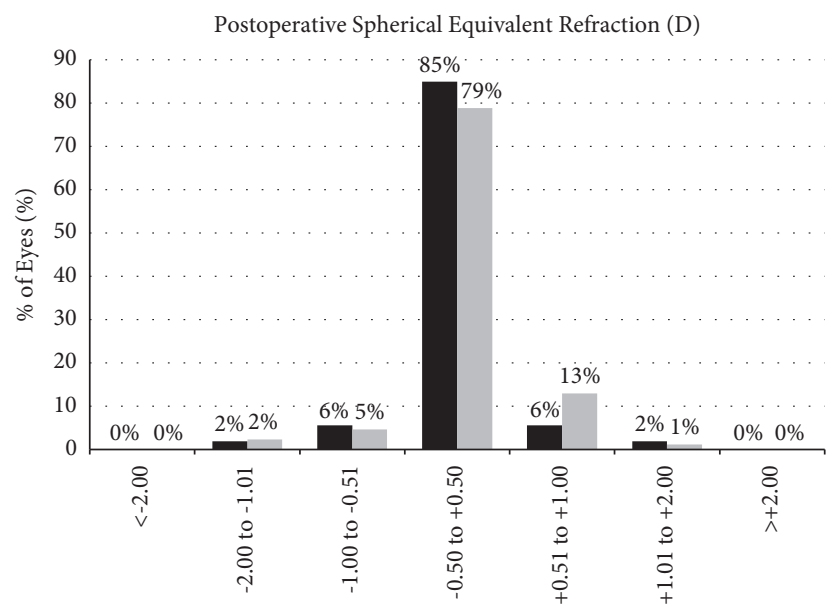

口 $\mathrm{SPT}$

non SPT

(d)

FIgURE 2: Continued. 


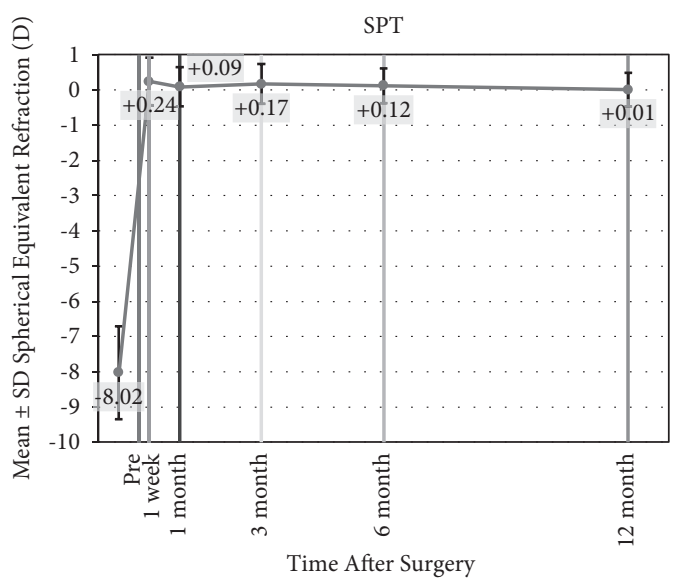

(e1)

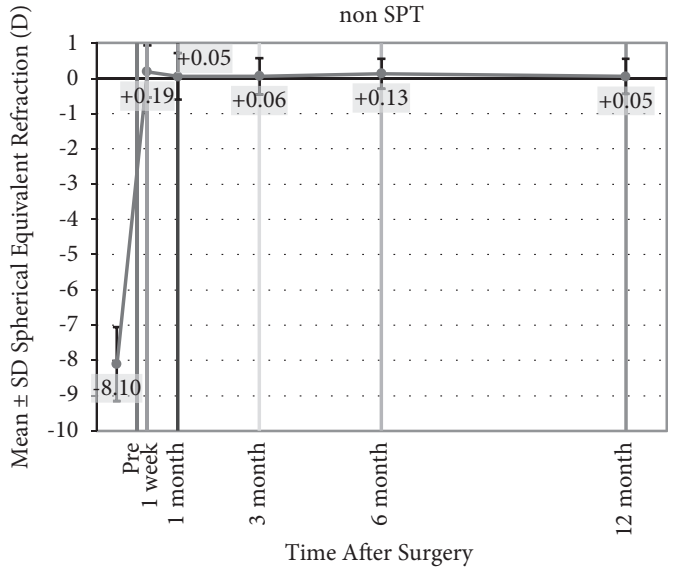

(e2)

(e)

Figure 2: (a) Efficacy of all surface laser ablation (ASLA) assisted by smart pulse technology (SPT group) versus ASLA without SPT (nonSTP group) 12 months postoperatively. (b) Change in corrected distance visual acuity. (c) Attempted vs. achieved spherical equivalent (SE) refraction in the SPT group and the non-SPT group. (d) SE refraction accuracy. (e) Preoperative, 1 week, and 1, 3, 6, and 12 months SE values in the SPT group and the non-SPT group.

TABLE 3: Changes of safety and efficacy index after 12 months in SPT group and non-SPT group ${ }^{\mathrm{a}}$.

\begin{tabular}{lccccc}
\hline Time & \multicolumn{3}{c}{ Safety index } & & \multicolumn{2}{c}{ Efficacy index } \\
& SPT group & Non-SPT group & $P$ & SPT group & Non-SPT group \\
\hline 7 days & $0.81 \pm 0.17$ & $0.71 \pm 0.17$ & $<0.001^{\mathrm{b}}$ & $0.79 \pm 0.22$ & $0.65 \pm 0.17$ \\
1 month & $0.99 \pm 0.08$ & $0.91 \pm 0.14$ & $<0.001^{\mathrm{b}}$ & $0.98 \pm 0.13$ & $0.84 \pm 0.24$ \\
3 months & $1.07 \pm 0.11$ & $1.02 \pm 0.13$ & $0.033^{\mathrm{b}}$ & $1.09 \pm 0.18$ & $0.99 \pm 0.19$ \\
6 months & $1.08 \pm 0.11$ & $1.07 \pm 0.12$ & 0.477 & $1.08 \pm 0.15$ & $0.99 \pm 0.18$ \\
12 months & $1.10 \pm 0.12$ & $1.04 \pm 0.11$ & $0.004^{\mathrm{b}}$ & $1.09 \pm 0.14$ & $0.001^{\mathrm{b}}$ \\
\hline
\end{tabular}

$\mathrm{SPT}=$ smart pulse technology. Safety index = postoperative mean CDVA/preoperative mean CDVA. Efficacy index = postoperative mean UDVA/preoperative mean CDVA. ${ }^{a}$ Values are presented as mean \pm standard deviation. ${ }^{\mathrm{b}}$ Significant difference between the SPT and non-SPT groups (Student's $t$-test/ Mann-Whitney $U$-test).
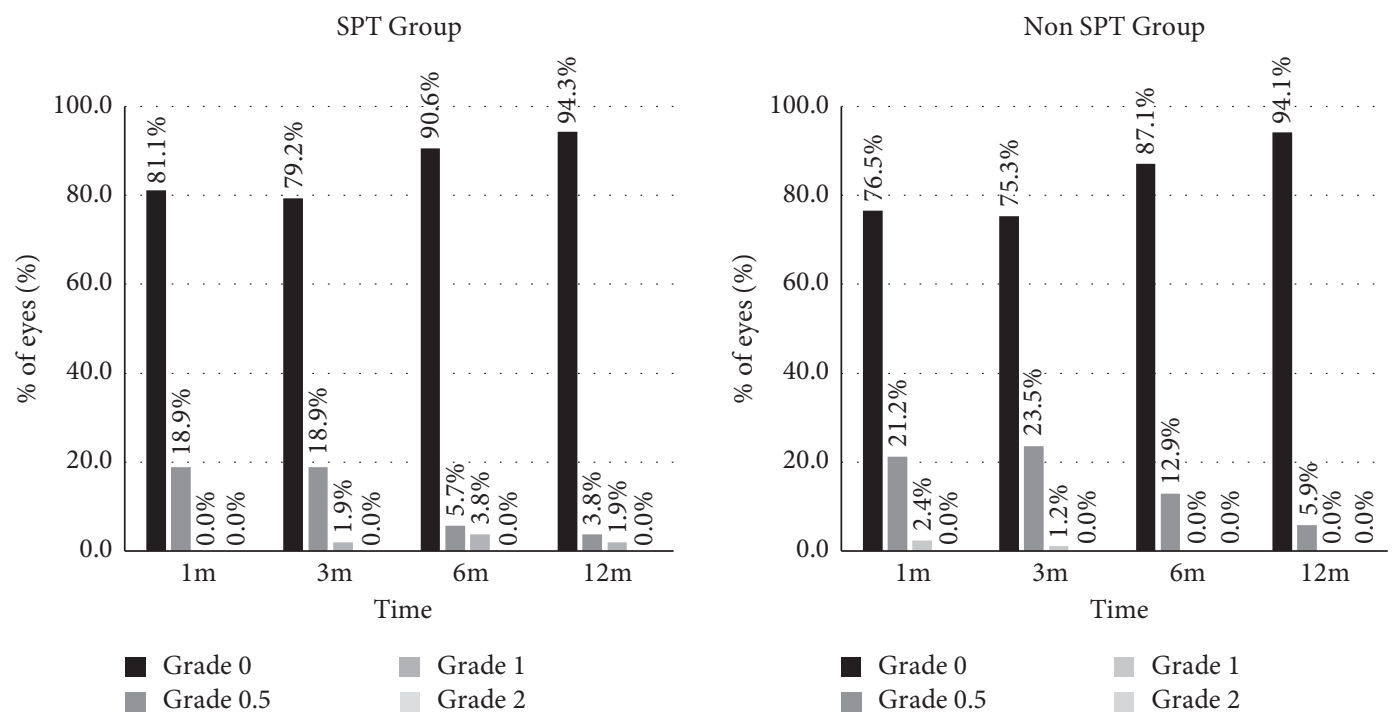

FIGURE 3: Variable occurrence rate of haze after SPT or non-SPT. SPT = smart pulse technology.

postoperatively were similar. More than $90 \%$ of eyes were within $1.0 \mathrm{D}$ at the 1-year follow-up which may provide an encouraging basis for the application of ASLA to patients with high myopia.
The proliferation of fibroblast and corneal turbidity would be reduced due to a smooth stromal surface [24-27]; as a consequence, haze in the SPT group theoretically was smaller than that in the non-SPT one. In Aslanides' and 
TABLE 4: Anterior corneal aberration values ${ }^{\mathrm{a}}$

\begin{tabular}{lccccc}
\hline Parameter & Group & Preoperative & After 12 months & $P$ & $\triangle($ 12-month postoperative vs. preoperative $)$ \\
\hline \multirow{3}{*}{ RMS HOA } & SPT group & $0.35 \pm 0.09$ & $0.89 \pm 0.24$ & $<0.001^{\mathrm{c}}$ & $0.54 \pm 0.25$ \\
& Non-SPT group & $0.38 \pm 0.10$ & $1.03 \pm 0.36$ & $<0.001^{\mathrm{c}}$ & $0.65 \pm 0.39$ \\
& $P^{*}$ & 0.069 & 0.059 & & 0.238 \\
\hline \multirow{3}{*}{ Spherical aberration } & SPT group & $0.25 \pm 0.09$ & $0.73 \pm 0.19$ & $<0.001^{\mathrm{c}}$ & $0.48 \pm 0.20$ \\
& Non-SPT group & $0.22 \pm 0.08$ & $0.77 \pm 0.21$ & $<0.001^{\mathrm{c}}$ & $0.56 \pm 0.22$ \\
& $P^{*}$ & $0.024^{\mathrm{b}}$ & 0.461 & & 0.091 \\
Coma $0^{\circ}$ & SPT group & $-0.05 \pm 0.10$ & $-0.29 \pm 0.26$ & $<0.001^{\mathrm{c}}$ & $-0.24 \pm 0.28$ \\
& Non-SPT group & $-0.11 \pm 0.10$ & $-0.31 \pm 0.39$ & $<0.001^{\mathrm{c}}$ & $-0.21 \pm 0.39$ \\
\hline \multirow{3}{*}{ Coma $90^{\circ}$} & $P^{*}$ & $0.001^{\mathrm{b}}$ & 0.840 & & 0.231 \\
& SPT group & $-0.00 \pm 0.15$ & $-0.15 \pm 0.42$ & $0.012^{\mathrm{c}}$ & $-0.15 \pm 0.39$ \\
\hline & Non-SPT group & $-0.04 \pm 0.20$ & $-0.40 \pm 0.51$ & $<0.001^{\mathrm{c}}$ & $-0.36 \pm 0.47$ \\
\hline
\end{tabular}

RMS HOA = root mean square higher order aberrations; SPT $=$ smart pulse technology. ${ }^{*}$ Compared with the SPT group and non-SPT group. ${ }^{\mathrm{a}}$ Values are presented as mean \pm standard deviation. ${ }^{b}$ Significant difference between the SPT and non-SPT groups (Student's $t$-test/Mann-Whitney $U$-test). ${ }^{c}$ Significant difference between preoperative and postoperative (Student's $t$-test/Wilcoxon test).

Vinciguerra's study $[6,10]$, there was no statistically difference between the SPT group and non-SPT group, which was similar to our results. Haze occurred in more than $20 \%$ of eyes in both groups at 1 month and 3 months after surgery which may be seem larger than the occurrence in previous research, but most of them were 0.5 level that barely affected vision and were gradually decreased and disappeared after the use of $0.1 \%$ fluorometholone eye drops. We consider that the main reason for this result is that haze is more likely to occur after correction of high myopia [26-28]. There were other objective factors which may also affect the results, such as the slight difference of clinician judgment for haze's corneal grade and patient's compliance.

Previous studies have reported that an increase in RMS HOAs of the anterior cornea was linearly correlated with the preoperative spherical equivalent and the central corneal ablation depth according to these results [29-32]. Juhasz et al. [30] found that RMS HOA values showed a 2.4 -fold growth when the ablation depth is $>77 \mathrm{um}$. In our study, RMS HOAs in both groups were also significantly increased about 2.5 3 times postoperatively, and the ablation depth was about $160 \sim 170 \mu \mathrm{m}$. The diopter of myopia and the ablation depth should be considered as important factors for increased HOAs. We expected that the introduction of SPT could reduce the postoperative spherical aberration by reducing the roughness of the cutting surface, but SPT did not seem to make an impact on the results in our study.

The results of horizontal coma (coma $0^{\circ}$ ) had significant difference between preoperation and postoperation both in the SPT and non-SPT groups, nor was there any significant difference between the two groups. However, the postoperative results of vertical coma (coma $90^{\circ}$ ) in both groups were significantly larger than the preoperative results, and the increase of coma $90^{\circ}$ in the SPT group was significantly less than that in non-SPT group. We have several theories about this outcome. On the one hand, higher order aberration effects (spherical aberration and coma) are mainly derived from the edge effect, that is, the change of local curvature from the optical region to the transition region and from the transition region to the untreated corneal region [33]. In normal mode, when the laser
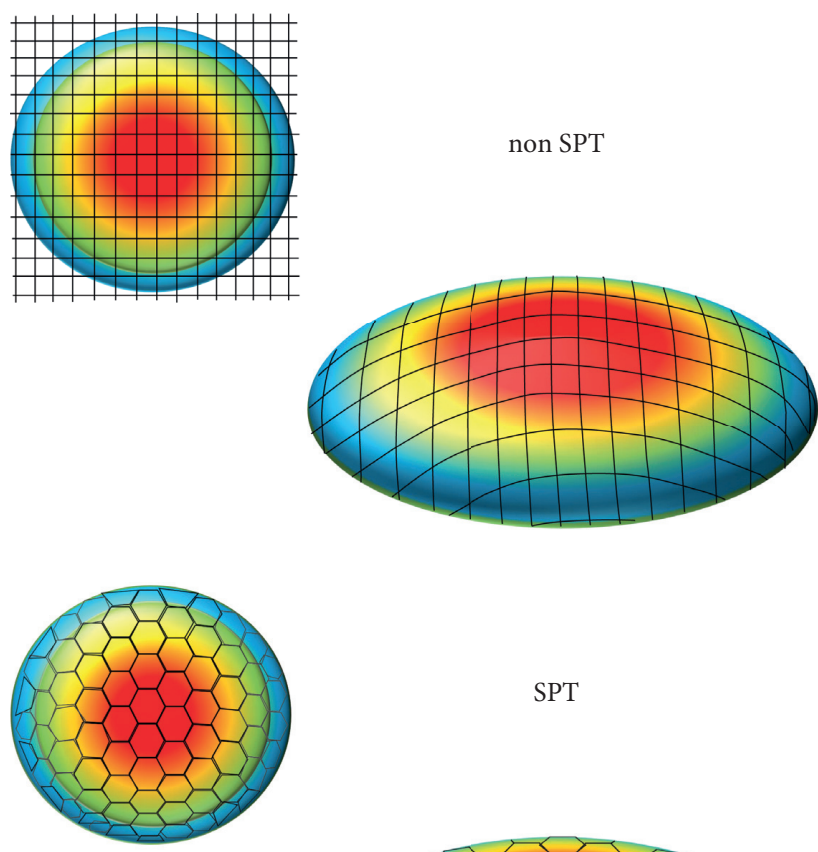

SPT

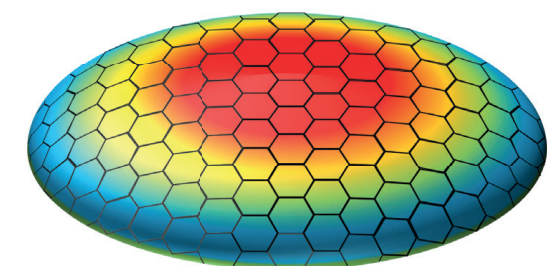

FIGURE 4: In the mode of non-SPT, the closer to the corneal periphery area is, the greater the energy loss is. While in the SPT mode, the energy loss is relatively reduced. SPT $=$ smart pulse technology.

ablation is near the periphery of the cornea, the ablating energy per unit area is lost due to the increase of the spot area [34], a phenomenon known as "the cosine effect" (Figure 4). In addition, the difference in the radius of local curvature between the upper and lower corneal areas leads to the difference in the energy loss of the surrounding area between the upper and lower corresponding region. As a result, the vertical coma 
would be inducted by the difference of local corneal ablation between the upper and lower parts. However, the improvement by SPT on the pattern and arrangement of laser spot could reduce the loss of laser energy which may make the energy per ablating area more uniform. In this mode, the ablating inhomogeneity caused by the difference of corneal curvature between the upper and lower areas may be relatively reduced, so that the increase of the asymmetry between the upper and lower parts may be reduced and the induction of vertical coma may be relatively reduced vs. non-SPT. On the other hand, since the distribution of corneal epithelium is asymmetric, the upward side is thick and the downward side is thin, and the difference in corneal remodeling process between the upper and lower areas may introduce more asymmetry and lead to the increase of coma $90^{\circ}$. However, SPT can make epithelial healing and remodeling more rapid and uniform by reducing the roughness of the corneal stromal surface, which may also reduce the additional coma introduced by the difference in thickness of the upper and lower corneal epithelium requiring remodeling.

More patients and longer follow-up are the next focus of this study.

\section{Conclusions}

Both SPT and non-SPT aberration-free ASLA techniques showed favorable safety, effectiveness, and predictability within 12 months for high myopia. ASLA using SPT might have potential advantages in the long-term visual quality.

\section{Data Availability}

The data are available from the corresponding author upon reasonable request.

\section{Disclosure}

$\mathrm{XH}-\mathrm{D}$ and JZ contributed equally to this work.

\section{Conflicts of Interest}

The authors declare no conflicts of interest.

\section{Authors' Contributions}

SH-C, XH-D, and JZ conceptualized and designed the study; XH-D, MS, WJ-C, and SZ collected data; SH-C, XH-D, JZ, MS, and Aslanides IM analyzed and interpreted the data; $\mathrm{XH}-\mathrm{D}$ wrote the manuscript; $\mathrm{SH}-\mathrm{C}$, Aslanides IM, XH-D, JZ, MS, and QM-W critically revised the manuscript; $\mathrm{XH}-\mathrm{D}$ and JZ were responsible for statistical expertise; $\mathrm{SH}-\mathrm{C}$, JZ, and QM-W provided administrative, technical, or material support; SH-C supervised the study.

\section{References}

[1] J. L. Alio, M. M. Ismael, and A. Artola, "Laser epithelium removal before photorefractive keratectomy," Journal of Refractive Surgery, vol. 9, no. 5, p. 395, 1993.

[2] T. E. Clinch, M. Moshirfar, J. R. Weis, C. S. Ahn, C. B. Hutchinson, and J. H. Jeffrey, "Comparison of mechanical and transepithelial debridement during photorefractive keratectomy," Ophthalmology, vol. 106, no. 3, pp. 483-489, 1999.

[3] A. Fadlallah, D. Fahed, K. Khalil et al., "Transepithelial photorefractive keratectomy: clinical results," Journal of Cataract and Refractive Surgery, vol. 37, no. 10, pp. 1852-1857, 2011.

[4] I. Aslanides, P. Padroni, S. Arba-Mosquera, and A. Mukherjee, "Comparison of single-step reverse transepithelial all-surface laser ablation (ASLA) to alcohol-assisted photorefractive keratectomy," Clinical Ophthalmology, vol. 6, pp. 973-980, 2012.

[5] I. Aslanides, P. Georgoudis, V. Selimis, and A. Mukherjee, "Single-step transepithelial ASLA (SCHWIND) with mitomycin-C for the correction of high myopia: long term followup," Clinical Ophthalmology, vol. 9, pp. 33-41, 2014.

[6] I. M. Aslanides and G. D. Kymionis, "Trans advanced surface laser ablation (TransPRK) outcomes using SmartPulseTechnology," Contact Lens and Anterior Eye, vol. 40, no. 1, pp. 42-46, 2017.

[7] A.-M. Soheil, S.-J. Saeed, and S. M. Ali, "Efficacy and safety of transepithelial photorefractive keratectomy," Journal of Cataract \& Refractive Surgery, vol. 44, no. 10, pp. 1267-1279, 2018.

[8] A. Mukherjee, A. Ioannides, and I. Aslanides, "Comparative evaluation of comfilcon A and senofilcon A bandage contact lenses after transepithelial photorefractive keratectomy," Journal of Optometry, vol. 8, no. 1, pp. 27-32, 2015.

[9] D. T. C. Lin, S. P. Holland, S. Verma, J. Hogden, and S. ArbaMosquera, "Postoperative corneal asphericity in low, moderate, and high myopic eyes after transepithelial PRK using a new pulse allocation," Journal of Refractive Surgery, vol. 33, no. 12, pp. 820-826, 2017.

[10] P. Vinciguerra, F. I. Camesasca, R. Arba-Mosquera, I. Torres, E. Morenghi, and J. B. Randleman, "Advanced surface ablation with a new software for the reduction of ablation irregularities," Journal of Refractive Surgery (Thorofare, N.J: 1995), vol. 33, no. 2, pp. 89-95, 2017.

[11] F. E. Fantes, K. D. Hanna, and G. O. Waring, "Wound healing after excimer laser keratomileusis (photorefractive keratectomy) in monkeys," Archives of Ophthalmology, vol. 108, no. 5, pp. 665-675, 1990.

[12] L. He and E. E. Manche, "Prospective randomized contralateral eye evaluation of subjective quality of vision after wavefront-guided or wavefront-optimized photorefractive keratectomy," Journal of Refractive Surgery, vol. 30, no. 1, pp. 6-12, 2014.

[13] A.-M. Soheil, S.-J. Saeed, and S. Bahram, "Single-step transepithelial photorefractive keratectomy in myopia and astigmatism: 18-month follow-up," Journal of Cataract \& Refractive Surgery, vol. 42, no. 11, pp. 1570-1578, 2016.

[14] R. Antonios, M. Abdul Fattah, S. Arba Mosquera, B. H. Abiad, K. Sleiman, and S. T Awwad, "Single-step transepithelial versus alcohol-assisted photorefractive keratectomy in the treatment of high myopia: a comparative evaluation over 12 months," British Journal of Ophthalmology, vol. 101, no. 8, pp. 1106-1112, 2017.

[15] L. Xi, C. Zhang, and Y. He, "Clinical outcomes of Transepithelial photorefractive keratectomy to treat low to moderate myopic astigmatism," BMC Ophthalmology, vol. 18, no. 1, p. 115, 2018.

[16] I. Jun, D. S. Kang, Y. Sung et al., "Comparison of clinical outcomes between wavefront-optimized versus corneal wavefront-guided transepithelial photorefractive keratectomy 
for myopic astigmatism," Journal of Cataract and Refractive Surgery, vol. 43, no. 2, pp. 174-182, 2017.

[17] S. Serrao and M. Lombardo, "Corneal epithelial healing after photorefractive keratectomy: analytical study," Journal of Cataract and Refractive Surgery, vol. 31, no. 5, pp. 930-937, 2005.

[18] M. Camellin and S Arba Mosquera, "Simultaneous aspheric wavefront-guided transepithelial photorefractive keratectomy and phototherapeutic keratectomy to correct aberrations and refractive errors after corneal surgery," Journal of Cataract and Refractive Surgery, vol. 36, no. 7, pp. 1173-1180, 2010.

[19] E. Anitua, F. Muruzabal, I. Alcalde, J. Merayo-Lloves, and G. Orive, "Plasma rich in growth factors (PRGF-Endoret) stimulates corneal wound healing and reduces haze formation after PRK surgery," Experimental Eye Research, vol. 115, pp. 153-161, 2013.

[20] C. A. Gauthier, D. Epstein, and B. A. Holden, "Epithelial alterations following photorefractive keratectomy for myopia," Journal of Refractive Surgery, vol. 11, no. 12, pp. 113-118, 1995.

[21] T. Moller-Pedersen, H. D. Cavanagh, and W. M. Petroll, "Stromal wound healing explains refractive instability and haze development after photorefractive keratectomy: a 1-year confocal microscopic study," Ophthalmology, vol. 107, no. 7, pp. 1235-1245, 2000.

[22] S. E. Wilson, R. R. Mohan, and J. W. Hong, "The wound healing response after laser in situ keratomileusis and photorefractive keratectomy," Archives of Ophthalmology, vol. 119, no. 6, pp. 889-896, 2001.

[23] J. C. Erie, "Corneal wound healing after photorefractive keratectomy: a 3-year confocal microscopy study," Transactions of the American Ophthalmological Society, vol. 101, pp. 293-333, 2003.

[24] M. V. Netto, R. R. Mohan, S. Sinha, A. Sharma, W. Dupps, and S. E. Wilson, "Stromal haze, myofibroblasts, and surface irregularity after PRK," Experimental Eye Research, vol. 82, no. 5, pp. 788-797, 2006.

[25] P. Vinciguerra, M. Azzolini, P. Airaghi, P. Radice, and V. De Molfetta, "Effect of decreasing surface and interface irregularities after photorefractive keratectomy and laser in situ keratomileusis on optical and functional outcomes," Journal of Refractive Surgery (Thorofare, N.J: 1995), vol. 14, no. 2, pp. S199-S203, 1998.

[26] S. E. Wilson, "Corneal myofibroblast biology and pathobiology: generation, persistence, and transparency," Experimental Eye Research, vol. 99, no. 1, pp. 78-88, 2012.

[27] F. W. De Medeiros and R. Mohan Rajiv, "Haze development after photorefractive keratectomy: mechanical vs. ethanol epithelial removal in rabbits," Journal of Refractive Surgery, vol. 24, no. 9, pp. 923-927, 2008.

[28] M. V. Netto, R. R. Mohan, S. Sinha, A. Sharma, P. C. Gupta, and S. E. Wilson, "Effect of prophylactic and therapeutic mitomycin $\mathrm{C}$ on corneal apoptosis, cellular proliferation, haze, and long-term keratocyte density in rabbits," Journal of Refractive Surgery, vol. 22, no. 6, pp. 562-574, 2006.

[29] L. Xi, "Wavefront properties of the anterior and posterior corneal surface after transepithelial photorefractive keratectomy in myopia," Experimental and therapeutic medicine, vol. 19, no. 2, pp. 1183-1188, 2020.

[30] E. Juhasz, K. Kranitz, G. L. Sandor, A. Gyenes, G. Toth, and Z. Z. Nagy, "Wavefront properties of the anterior and posterior corneal surface after photorefractive keratectomy," Cornea, vol. 33, no. 2, pp. 172-176, 2014.
[31] S. Serrao, G. Lombardo, P. Ducoli, and M. Lombardo, "Longterm corneal wavefront aberration variations after photorefractive keratectomy for myopia and myopic astigmatism," Journal of Cataract \& Refractive Surgery, vol. 37, no. 9, pp. 1655-1666, 2011.

[32] S.-B. Lee, B.-S. Hwang, and J. Lee, "Effects of decentration of photorefractive keratectomy on the induction of higher order wavefront aberrations," Journal of Refractive Surgery, vol. 26, no. 10, pp. 731-743, 2010.

[33] A.-M. Samuel and D. De Ortueta, "Analysis of optimized profiles for "aberration-free" refractive surgery," Ophthalmic and Physiological Optics, vol. 29, no. 5, pp. 535-548, 2009.

[34] C. R. Munnerlyn, S. J. Koons, and J. Marshall, "Photorefractive keratectomy: a technique for laser refractive surgery," Journal of Cataract \& Refractive Surgery, vol. 14, no. 1, pp. $46-52,1988$ 